\title{
BMJ Open Complications in breast augmentation with textured versus smooth breast implants: a systematic review protocol
}

\author{
Chenglong Wang, ${ }^{1}$ Jie Luan, ${ }^{1}$ Adriana C Panayi, ${ }^{2}$ Dennis P Orgill, ${ }^{2}$ Minqiang Xin ${ }^{1}$
}

To cite: Wang C, Luan J, Panayi AC, et al. Complications in breast augmentation with textured versus smooth breast implants: a systematic review protocol. BMJ Open 2018;8:e020671. doi:10.1136/ bmjopen-2017-020671

- Prepublication history and additional material for this paper are available online. To view these files, please visit the journal online (http://dx.doi. org/10.1136/bmjopen-2017020671).

Received 26 November 2017 Revised 31 January 2018 Accepted 8 February 2018
Check for updates

${ }^{1}$ Department of Aesthetic and Reconstructive Breast Surgery, Plastic Surgery Hospital, Peking Union Medical College, Chinese Academy of Medical Sciences, Beijing, China

${ }^{2}$ Division of Plastic Surgery, Harvard Medical School, Brigham and Women's Hospital, Boston, Massachusetts, USA

Correspondence to Dr Minqiang Xin; doctorshin@126.com

\section{ABSTRACT}

Introduction Breast augmentation is one of the most popular aesthetic plastic surgeries worldwide. There are various types of breast implants, and these can be categorised into different broad groups based on their content, shape or surface, to name a few. When looking at the surface of the shell, they can be categorised into two main kinds: textured and smooth implants. To our knowledge, a literature review and meta-analysis of the complications of these two types of implants when used for aesthetic breast augmentation has yet to be written. Methods and analysis The PubMed, EMBASE and Cochrane electronic databases will be searched from their inception to 10 ctober 2017. Only cohort studies, case series, case-control studies and randomised controlled trials will be included. Identification of the articles for inclusion will be carried out by two independent researchers, and data will be extracted from these studies for analysis. This protocol defines the inclusion and exclusion criteria, as well as the primary and secondary outcomes. Statistical data analysis will be conducted in Review Manager V.5.3 from Cochrane Collaboration. The methodological quality of the included studies will also be assessed.

Ethics and dissemination This review will analyse secondary data collected from studies which are not linked to any specific individual. Once completed, the conclusions of the review could prove to be a valuable resource for plastic surgeons to conduct aesthetic implant procedures. The review will be submitted for publication in a peerreviewed journal and presented at various national and international conferences.

PROSPERO registration number CRD42017078727.

\section{INTRODUCTION}

Ever since Cronin and Brauer first introduced the silicone breast implant, ${ }^{1}$ breast augmentation with prosthetic implants has become one of the most frequently performed aesthetic surgical procedures worldwide. ${ }^{2}$ Breast prostheses can be separated into two groups based on the surface of their shell: textured and smooth implants. Textured implants were more recently developed with the dual purpose of both stabilising the implant in the breast pocket and decreasing the rate of capsular contracture. ${ }^{3}$
Strengths and limitations of this study

The study findings will provide evidence for plastic surgeons to understand the different complications of textured and smooth breast implants.

- The review includes as many complications of breast implants as possible.

- The review will not include unpublished studies or those published in a language other than English. The quality of the primary studies to be included in this review may be a limiting factor due to the different study design.

Despite research supporting that textured implants have a lower incidence of capsular contracture, $^{4} 5$ this conclusion remains controversial. Many plastic surgeons prefer to use smooth implants as they find that contracture rates between textured and smooth implants are comparable. ${ }^{6-9}$ In addition to capsular contracture, breast implants have been associated with other complications, including infection, haematoma, seroma, rupture, malposition and ripple deformity. No consensus has yet to be reached on whether there is a significant difference in the aforementioned complication rates between textured and smooth implants. For example, double capsules ${ }^{10}$ and breast implant-associated anaplastic large cell lymphoma (BIA-ALCL) ${ }^{11}$ appear to be exclusively related to textured implants. Furthermore, there is evidence that rupturing is less prevalent with textured than smooth implants. ${ }^{12}$ Although systematic review articles comparing the use of textured and smooth implants have been conducted, these either focused solely on one type of complication, that is, the occurrence of capsular contracture, ${ }^{13}$ or on various complication types but when used in reconstructive, rather than aesthetic, breast surgery. $^{15}$

This review will help elucidate whether there is indeed a difference in complications, 
including but not limited to rupture, ripple deformity and rate of implant exchange.

Given that there are many other complications beyond capsular contracture, and a large proportion of breast augmentation procedures carried out are aesthetic, this review seeks to summarise and the currently available literature to provide a more comprehensive understanding of the complications associated with aesthetic breast augmentation using textured versus smooth implants. Conclusions from such a review aim to provide plastic surgeons with clearer information on the complications of textured and smooth implants, offering a suggestion as to which shell, if either, is superior.

\section{OBJECTIVE}

To conduct a systematic review exploring the complications of textured and smooth breast implants when used for aesthetic purposes that have been identified in the existing literature.

The complications of interest are:

1. Capsular contracture.

2. Double capsules.

3. Haematoma.

4. Infection.

5. Excessive waviness and/or rippling.

6. Implant rupture.

7. Reoperation rate.

8. BIA-ALCL.

9. Patient satisfaction.

\section{METHODOLOGY}

This systematic review was registered in PROSPERO (PROSPERO 2017: CRD42017078727) and will be conducted based on the recommendations provided by the Cochrane Handbook for Systematic Reviews and reported in line with the Preferred Reporting Items for Systematic Reviews and Meta-Analyses statement. ${ }^{16}$

\section{Criteria for selecting studies}

The inclusion criteria for this review and meta-analysis are as follows.

\section{Types of studies/material}

Studies eligible for inclusion are case series, cohort studies, case-control studies and randomised controlled trials which mention at least one of the predefined primary complications of interest. Studies of implants used in a reconstructive setting, unpublished trials and reports, studies that fail to provide an indication of the type of breast implant, case reports, duplicate studies and studies that do not provide the original data, such as systematic reviews and meta-analyses, will be excluded.

\section{Types of participants}

Female patients undergoing aesthetic breast surgery using bilateral breast implants.
Types of interventions

Aesthetic surgical interventions using smooth or textured implants will be considered. The intervention should have been solely performed for aesthetic purposes. Reconstructions following oncological or trauma (such as amputations or burns) surgery will be excluded.

\section{Outcomes}

\section{Primary outcomes}

1. Capsular contracture: based on (1) palpation using the Baker scale (grades $\square$ and $\square$ defined as capsular contracture); (2) patient subjective assessment; (3) relative applanation tonometry. ${ }^{14}$

2. Haematoma: presence of significantly greater than anticipated bruising, swelling and firmness of the breast or surgical exploration revealing the presence of excessive blood surrounding the implant. ${ }^{17}$

3. Infection: presence of abnormal swelling, tenderness, erythema and fever resolving with antibiotics or requiring explantation. ${ }^{17}$

4. Excessive waviness and/or rippling: deemed excessive either due to the requirement of surgical revision, or due to patients' self-reporting the deformity as problematic.

5. Rupture of implants: clinically confirmed at the time of explantation.

6. Anaplastic large cell lymphoma.

7. Reoperation rates: number of surgeries required to achieve a successful outcome.

8. Patient satisfaction (BREAST-Q scale for augmentation).

\section{Secondary outcome}

Determine the optimal implant for aesthetic breast surgery.

\section{SEARCH METHODOLOGY FOR IDENTIFICATION OF STUDIES Electronic searches}

The PubMed, EMBASE and Cochrane electronic databases will be screened from their inception to 1 October 2017.

\section{Search terms and keywords}

The following keywords will be used for the database search: aesthetic plastic surgery, aesthetic breast surgery, cosmetic plastic surgery, cosmetic breast surgery, breast augmentation, mammaplasty, breast implant, breast prosthesis, breast prostheses, mammary implant, smooth implant, textured implant, saline implant, silicone implant. A free-text search or a MeSH search will be performed when appropriate and terms can be combined using Boolean operators (online supplementary table). The language will be restricted to English and the search format will be tailored to the appropriate syntax of each database.

Box 1 outlines the inclusion and exclusion criteria. 
Box 1 Inclusion and exclusion criteria for study selection

Inclusion criteria
Cohort studies, case series, case-control studies and randomised
controlled trials.
Prospective and retrospective studies.
Patients who underwent aesthetic breast surgery using bilateral
breast implants.
Female patients of any age.
Aesthetic surgical interventions using smooth or textured implants.
At least one primary outcome reported.
Exclusion criteria
Unpublished trials and reports, case reports, duplicate studies,
cost-effectiveness studies and studies that do not provide the origi-
nal data such as systematic reviews, meta-analyses, editorials, dis-
cussions, commentaries and letters.
Non-English language studies.
Studies with no data on complications.
Studies focusing on reconstructive surgery following oncological or
trauma surgery.
Studies that did not specify the number of patients.

\section{Identification and selection of articles}

The title, citation and abstract of all studies identified through the electronic search will be tabulated in Microsoft Excel and any duplicates will be removed. Two independent reviewers will then evaluate the references, eliminating ineligible studies first, based on titles and abstracts, and finally based on full text. Consensus on disagreement of inclusion would ideally be reached on discussion, or failing that through consultation of a third author.

\section{Data extraction and quality assessment}

Two independent reviewers will carry out the data extraction using a pre-established standardised form. The data will be collated as follows:

- Reference (first author, year of publication, country).

- Type of study.

- Sample size.

- Patient mean age.

- Length of follow-up.

- Follow-up rate.

- Implant type.

- Implant pocket placement.

- Incision type.

\section{Primary outcomes as outlined above}

The Jadad score will be used to assess the methodological quality of any randomised controlled trials, ${ }^{18}$ and the methodological index for non-randomised studies instrument for the quality of non-randomised studies. ${ }^{19}$

\section{Risk of bias assessment}

Risk of bias will be assessed independently by CLW and ACP using the Cochrane risk of bias tool for randomised and non-randomised studies. ${ }^{20}$ Disagreements will be solved by discussion, and MQX will adjudicate unresolved disagreements.

\section{Confidence in cumulative evidence}

The Grading Quality of Evidence and Strength of Recommendations will be used to assess the quality and strength of the body of evidence. ${ }^{21}$

\section{Data synthesis and statistical analysis}

The primary and secondary outcomes, as defined above, will be summarised in descriptive or numeric form as appropriate. Statistical analysis will be conducted using Review Manager V.5.3 from Cochrane Collaboration.

\section{Ethics and dissemination}

The review and meta-analysis will provide plastic surgeons a summary and comparison of the different complications associated with textured and smooth breast implants. The aim is to publish the article in the English language in a peer-reviewed journal and present the evidence at national and international conferences.

\section{CONCLUSION}

Through the systematic review we aim to evaluate the currently available evidence in order to summarise and compare the complications seen with textured and smooth breast implants used in aesthetic breast surgery. This review could prove to be a valuable tool for plastic surgeons, providing a comparison of the complications and success rate of two types of prosthesis and potentially assisting surgeons in their choice of prosthesis in clinical practice.

Contributors MX and CW had the original idea of this work. CW wrote the first draft of the protocol. CW and ACP designed the search strategies. JL proposed some important advice for the study design and revision. ACP revised the language of the draft. DPO aided in developing the research questions. All authors critically revised the draft of the manuscript and approved its final version.

Funding This research received no specific grant from any funding agency in the public, commercial or not-for-profit sectors.

Competing interests None declared.

Patient consent Not required.

Provenance and peer review Not commissioned; externally peer reviewed.

Open Access This is an Open Access article distributed in accordance with the Creative Commons Attribution Non Commercial (CC BY-NC 4.0) license, which permits others to distribute, remix, adapt, build upon this work non-commercially, and license their derivative works on different terms, provided the original work is properly cited and the use is non-commercial. See: http://creativecommons.org/ licenses/by-nc/4.0/

(c) Article author(s) (or their employer(s) unless otherwise stated in the text of the article) 2018. All rights reserved. No commercial use is permitted unless otherwise expressly granted.

\section{REFERENCES}

1. Cronin TD, Brauer RO. Augmentation mammaplasty. Surg Clin North Am 1971;51:441-52.

2. Heidekrueger PI, Sinno S, Hidalgo DA, et al. Current trends in breast augmentation: an international analysis. Aesthet Surg $J$ 2018;38:133-48. 
3. Efanov JI, Giot JP, Fernandez J, et al. Breast-implant texturing associated with delamination of capsular layers: A histological analysis of the double capsule phenomenon. Ann Chir Plast Esthet 2017;62:196-201.

4. Pollock H. Breast capsular contracture: a retrospective study of textured versus smooth silicone implants. Plast Reconstr Surg 1993;91:404-7.

5. Spear SL, Elmaraghy M, Hess C. Textured-surface saline-filled silicone breast implants for augmentation mammaplasty. Plast Reconstr Surg 2000;105:1553-4.

6. Becker H, Springer R. Prevention of capsular contracture. Plast Reconstr Surg 1999:103:1766-8.

7. Biggs TM. Augmentation mammaplasty: a comparative analysis. Plast Reconstr Surg 1999;103:1761-2.

8. Fagrell D, Berggren A, Tarpila E. Capsular contracture around salinefilled fine textured and smooth mammary implants: a prospective 7.5-year follow-up. Plast Reconstr Surg 2001;108:2108-12.

9. Handel N, Jensen JA, Black Q, et al. The fate of breast implants: a critical analysis of complications and outcomes. Plast Reconstr Surg 1995;96:1521-33.

10. Clemens MW, Nava MB, Rocco N, et al. Understanding rare adverse sequelae of breast implants: anaplastic large-cell lymphoma, late seromas, and double capsules. Gland Surg 2017;6:169-84.

11. O'Neill AC, Zhong T, Hofer SOP. Implications of Breast ImplantAssociated Anaplastic Large Cell Lymphoma (BIA-ALCL) for Breast Cancer Reconstruction: An Update for Surgical Oncologists. Ann Surg Oncol 2017;24:3174-9.

12. Haws MJ, Alizadeh K, Kaufman DL. Sientra primary and revision augmentation rupture trending and analysis with magnetic resonance imaging. Aesthet Surg J 2015;35(Suppl 1):S33-42.
13. Liu X, Zhou L, Pan F, et al. Comparison of the postoperative incidence rate of capsular contracture among different breast implants: a cumulative meta-analysis. PLoS One 2015;10:e0116071.

14. Wong CH, Samuel M, Tan BK, et al. Capsular contracture in subglandular breast augmentation with textured versus smooth breast implants: a systematic review. Plast Reconstr Surg 2006;118:1224-36.

15. Rocco N, Rispoli C, Moja L, et al. Different types of implants for reconstructive breast surgery. Cochrane Database Syst Rev 2016;5:CD010895.

16. Moher D, Liberati A, Tetzlaff J, et al. Preferred reporting items for systematic reviews and meta-analyses: the PRISMA statement. Int $J$ Surg 2010;8:336-41.

17. Handel N, Cordray T, Gutierrez J, et al. A long-term study of outcomes, complications, and patient satisfaction with breast implants. Plast Reconstr Surg 2006;117:757-67.

18. Jadad AR, Moore RA, Carroll D, et al. Assessing the quality of reports of randomized clinical trials: is blinding necessary? Control Clin Trials 1996;17:1-12.

19. Slim K, Nini E, Forestier D, et al. Methodological index for nonrandomized studies (minors): development and validation of a new instrument. ANZ J Surg 2003;73:712-6.

20. Higgins JP, Altman DG, Gøtzsche PC, et al. The Cochrane Collaboration's tool for assessing risk of bias in randomised trials. BMJ 2011;343:d5928

21. Atkins D, Best D, Briss PA, et al. Grading quality of evidence and strength of recommendations. BMJ 2004;328:1490. 